\title{
The Rawdon-Smith illusion
}

\author{
WALT JESTEADT, DAVID M. GREEN, and CRAIG C. WIER \\ Laboratory of Psychophysics, Harvard University, Cambridge, Massachusetts 02138
}

\begin{abstract}
A loudness illusion is described which was originally reported by Rawdon-Smith and Grindley (1935). It is analogous to the Craik-O'Brien-Cornsweet brightness illusion. Procedures are described for generating and measuring the size of the effect, and data are presented showing factors that influence its magnitude. Other examples are discussed that suggest that this effect is a very general phenomenon.
\end{abstract}

Cornsweet (1970, p. 273) and O'Brien (1958) describe a brightness illusion associated with sharp spatial transitions in luminance. The first discussion of this illusion, however, appears in an unpublished thesis by Kenneth J. W. Craik (1940), whose notes on the subject were published posthumously (Sherwood, 1966). Craik indicated that his work was motivated in part by an attempt to produce a brightness illusion analogous to the loudness illusion reported by his colleague Rawdon-Smith (Rawdon-Smith $\&$ Grindley, 1935). To do this, he equated luminance with sound pressure level and spatial extent with time. The brightness illusion has subsequently received considerably more attention than the loudness illusion. The brightness illusion has been rediscovered several times and has gone through a series of refinements that maximize the size of the effect. It is now commonly referred to as the Craik-O'Brien illusion or the Craik-O'Brien-Cornsweet illusion. We recently rediscovered the loudness illusion and report here on procedures for generating and measuring it and on parameters that influence its magnitude. We have referred to it elsewhere as "an auditory analogue of the Craik-O'Brien-Cornsweet illusion" (Jesteadt, Green, \& Wier, 1976), but it seems more parsimonious and historically more accurate to call it the Rawdon-Smith illusion.

Rawdon-Smith and Grindley (1935) used a $2,000-\mathrm{Hz}$ sinusoid that fluctuated in intensity. It in-

This research was supported by a grant from the National Institutes of Health, U.S. Department of Health, Education, and Welfare. The first and third authors were supported by NIH postdoctoral fellowships. Preparation of this article was supported by the Boys Town Institute for Communication Disorders in Children. We wish to thank Charles Stromeyer for pointing out the existence of several important studies in the vision literature and Charles S. Watson for helpful comments on earlier drafts of the manuscript. Requests for reprints should be addressed to Walt Jesteadt, who is now at the Boys Town Institute for Communication Disorders in Children, 555 North 30th Street, Omaha, Nebraska 68131. Craig C. Wier is now at the Department of Speech and Hearing Seiences, University of Arizona, Tucson, Arizona 85721 creased rapidly by $1.7 \mathrm{~dB}$, then decreased slowly over an 8-sec interval until it reached the initial level. This pattern was repeated continuously. Four out of their six subjects detected the rapid increases in intensity, but not the slow decreases, and reported a sensation of increasing loudness. Reversing the direction of the transitions or changing their magnitude reduced the effect. We have listened to this stimulus configuration and do not find the effect strong. It is analogous to the visual stimulus configurations used by Craik and by O'Brien (1958). A stronger brightness illusion, and also a stronger loudness illusion, is produced using a configuration of the type reported by Cornsweet (1970). Examples of the auditory analogue, as it would appear on an oscilloscope, are shown in the left half of Figure 1. Subjects listening to this configuration with either a noise or sinusoidal
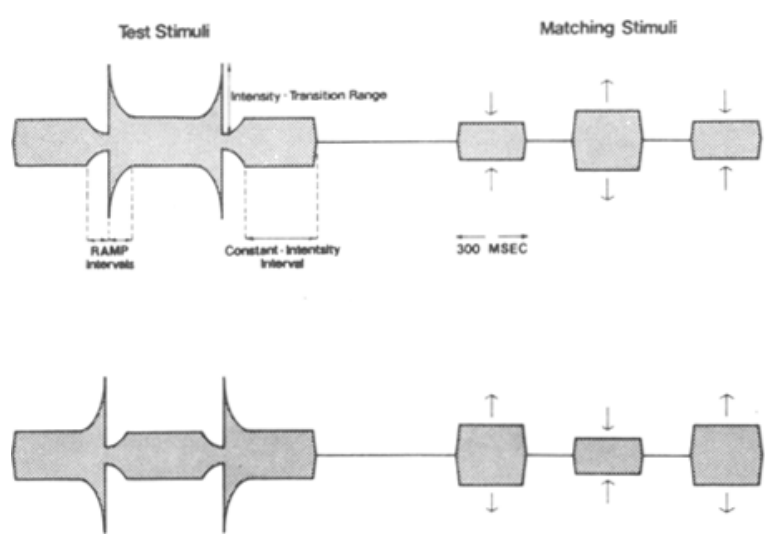

Figure 1. Summary of the matching paradigm. Envelopes of two forms of the test stimuli are shown on the left. In this example, the constant-intensity intervals are $300 \mathrm{msec}$, the ramp intervals are $100 \mathrm{msec}$, and the intensity-transition range is $20 \mathrm{~dB}$ from peak to trough. The subject's task was to adjust the relative intensities of the three intervals in the matching stimuli, shown on the right, to match the perceived intensities of the corresponding constant-intensity intervals of the test stimuli. Each adjustment response caused the intensities to change in all three intervals, as shown by the arrows, so that the overall intensity of the matching stimulus remained equal to that of the test stimulus. 
carrier report a sensation of alternating loud and soft intervals separated by very sharp transitions.

In the following sections, we report on two procedures used to measure the size of the illusion and on factors that influence its magnitude. We conclude with a discussion of other examples which suggest that the illusion is a general sensory phenomenon.

\section{EXPERIMENT 1: LOUDNESS-MATCHING PROCEDURE}

\section{Method}

Subjects. Two listeners with extensive experience in forcedchoice and adjustment experiments served as paid subjects. They were unaware of the physical nature of the auditory stimuli and were not told that the phenomenon being studied could be considered an illusion.

Apparatus. Stimulus configurations similar to the ones shown in Figure 1 were generated by passing an analogue signal from a Wavetek oscillator (Model 116) or an Eigenco noise generator through a Charybdis programmable attenuator. The attenuator was controlled by a Digital Equipment Corporation PDP-15 computer and was used to gate the signals on and of as well as to generate the intensity transitions shown in Figure 1. The transitions were produced by changing the attenuator in $.25-\mathrm{dB}$ steps at whatever rate was required to cover the specified range in the specified duration. The attenuator output was bandpass filtered to reduce onset and of fset clicks, passed through a manual attenuator to control overall level, and then through a power amplifier to a TDH-39 phone. The subject listened to the stimuli monaurally in a double-walled, sound-treated room.

Procedure. The matching procedure is shown in Figure 1. The test stimulus took one of two forms, shown in the left half of Figure 1. The matching stimulus, shown in the right half of Figure 1, consisted of three tone bursts (or three noise bursts if a noise carrier was used in the test stimulus). The duration of each of the bursts was equal to the duration of the corresponding constant-intensity interval in the test stimulus, and the durations of the intervals between bursts were equal to the durations of the transition intervals in the test stimulus. The constant-intensity intervals in the test stimuli and the corresponding tone or noise bursts in the matching stimuli were marked by lights.

All of the test stimuli were symmetrical in that the durations of the constant-intensity intervals were equal to one another, the ramp durations were equal to one another, and the intensity difference in decibels between the constant-intensity interval and the peak of the transition was equal to the difference between the constant-intensity interval and the trough. Intensity changes as a function of time were linear in decibels.

The test-stimulus parameters varied were (1) the intensitytransition range, or intensity difference from peak to trough, (2) the constant-intensity interval duration, (3) the duration of the ramp or slow transition, (4) the frequency or bandwidth of the carrier, and (5) the intensity of the carrier.

The subject heard one full cycle of the test stimulus, followed $600 \mathrm{msec}$ later by the matching stimulus. This sequence was repeated after a $1-\sec$ quiet interval and this continued until the adjustment was complete. The subject was instructed to adjust the relative intensities of the tone or noise bursts in the matching stimulus so that the relative loudness of these bursts equaled the relative loudness of the constant-intensity intervals within the test stimulus. The subject was told to ignore the transition intervals in the test stimulus, when the marker light was off, for purposes of making the loudness match.

The adjustments were made by pushing one of four buttons on a response panel in the sound-treated room. The buttons were interfaced to the computer and caused the intensity of the middle burst in the matching stimulus to change with respect to the intensity of the initial and final bursts, which were always equal to one another. These changes in intensity were produced by moving the middle burst in one direction and the initial and final burst in the other, so that the overall intensity of the three bursts remained centered on the intensity of the constant intervals in the test stimulus.' One pair of buttons caused a change of $\pm 2 \mathrm{~dB}$ in the difference between the intensities of the middle and the surrounding bursts. The other pair, used for fine adjustments, caused a change of $\pm 1 / 2 \mathrm{~dB}$.

The test and matching stimuli alternated until the subject pushed another button indicating he was satisfied with the match. The computer program then presented a new test stimulus and a matching stimulus with an initial intensity difference selected at random from the range of values between 5 and $-5 \mathrm{~dB}$ and another matching sequence was begun.

A subject typically made matches to two sets of two practice stimuli and 24 test stimuli in a 2 -h session. The test stimuli within a set differed only in intensity range. They all had the same constant-intensity duration, ramp durations, and carrier. Each of six intensity-transition ranges was presented four times in random order, twice in the form shown in the upper half of Figure 1 and twice in the form shown in the lower half. The use of the two forms and a number of intensity-transition ranges insured a wide range of matches and prevented the subjects from simply reproducing the final setting of the previous matching stimulus to achieve consistent matches.

\section{Results}

The standard stimulus configuration shown in Figure 1, with 300 -msec constant intensity intervals and 100 -msec ramp durations, was used with a number of different carriers. Data for sinusoidal carriers, shown in Figure 2, indicate that carrier frequency has no effect on the magnitude of the perceived difference in loudness. There is, however, a strong linear relation between the size of the intensitytransition range and the apparent difference in intensity.

The deviant point in Figure 2, for $250 \mathrm{~Hz}$ and $20-\mathrm{dB}$ range, illustrates one of the major problems

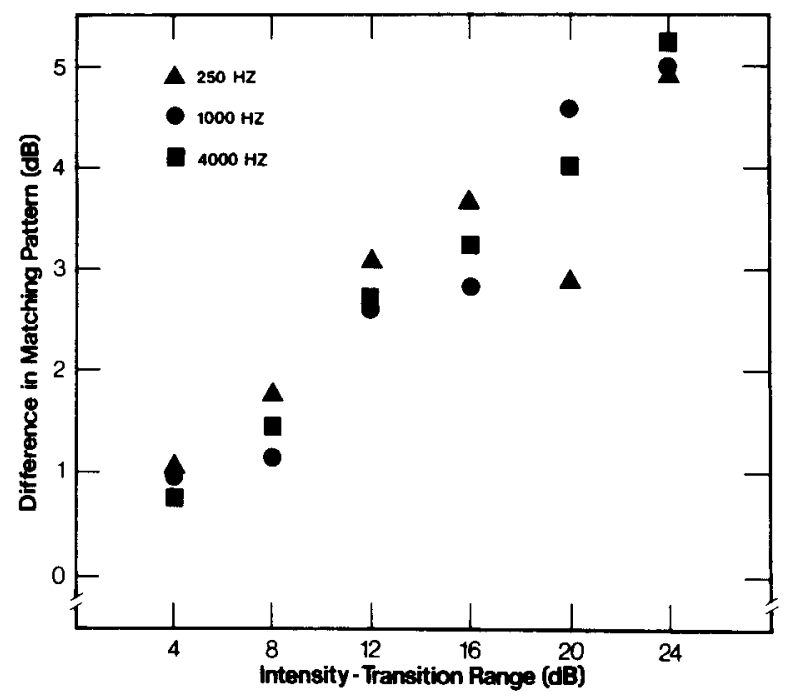

Figure 2. Perceived differences in intensity as a function of intensity-transition range for three carrier frequencies. Each point is the mean of four to six matches for each of the two subjects. 
with this procedure. The subjects occasionally arrived at matches that agreed with their other matches in magnitude but differed in sign. That is, instead of adjusting the matching pattern so that it was loudsoft-loud, they would adjust it so that it was softloud-soft. This "error" was particularly prevalent in sessions involving short constant intervals or short ramp intervals. The subjects' introspective reports indicated confusions of temporal order. We had no basis on which to reject these matches, however, and simply averaged them in. In a few cases where the variability in a set of matches was extremely high, we collected additional data. In most cases, the standard deviation for the four matches by a given subject was about $1 \mathrm{~dB}$, and the means of the matches for the two subjects were within $2 \mathrm{~dB}$ of one another.

In addition to varying carrier frequency, we also varied carrier intensity, using a 1,000- $\mathrm{Hz}, 30-\mathrm{dB}$ SPL carrier, and carrier bandwidth, using $500-1,500-\mathrm{Hz}$ and $0-10,000-\mathrm{Hz}, 70-\mathrm{dB}$ SPL noise. The results are summarized in Table 1 . The $1,000-\mathrm{Hz} 70-\mathrm{dB}$ SPL data from Figure 2 are included for comparison purposes. The differences, if any, are small. We conclude that the carrier or fine structure and the absolute level of the stimulus have little effect on the magnitude of the illusion, compared to variables such as intensity-transition range that govern the envelope shape. We therefore used a $1,000-\mathrm{Hz}$, 70-dB SPL carrier in all of the remaining conditions.

The effects of varying ramp duration for a stimulus with a 300 -msec constant-intensity interval are shown in Figure 3. Here we have taken advantage of the fact that the effect is generally linear as a function of intensity-transition range and have collapsed across that variable by fitting linear functions to the range data for each subject and evaluating those functions at an intensity-transition range of $20 \mathrm{~dB}$.

The effect of varying constant-intensity interval duration is shown in Figure 4 for two different ramp durations. Again, we have collapsed across intensity-

Table 1

Effects of Carrier Level and Carrier Bandwidth on the Perceived Difference in Loudness for Different Transition Ranges

\begin{tabular}{|c|c|c|c|c|c|c|c|}
\hline & & \multicolumn{6}{|c|}{ Intensity Range } \\
\hline \multicolumn{2}{|c|}{ Condition } & 4 & 8 & 12 & 16 & 20 & 24 \\
\hline 1,0 & 70 & .94 & 1.12 & 2.56 & 2.8 & 4.56 & 5.00 \\
\hline $1,000 \mathrm{~Hz}$ & $30 \mathrm{~d}$ & 1.69 & 1.62 & 3.50 & 4.94 & 5.56 & 5.31 \\
\hline $500-1,500 \mathrm{~Hz}$ & $70 \mathrm{~dB}$ SPL & .42 & 1.87 & 2.38 & 2.92 & 3.58 & 3.17 \\
\hline $0-1,000 \mathrm{~Hz}$ & $70 \mathrm{~dB}$ SPL & .18 & 1.75 & 2.42 & 4.16 & 4.34 & 5.25 \\
\hline
\end{tabular}

Note-In all conditions, the duration of the slow transition was $100 \mathrm{msec}$ and the duration of the constant-intensity interval was 300 msec. Each point is the mean of the adjustments for two subjects.

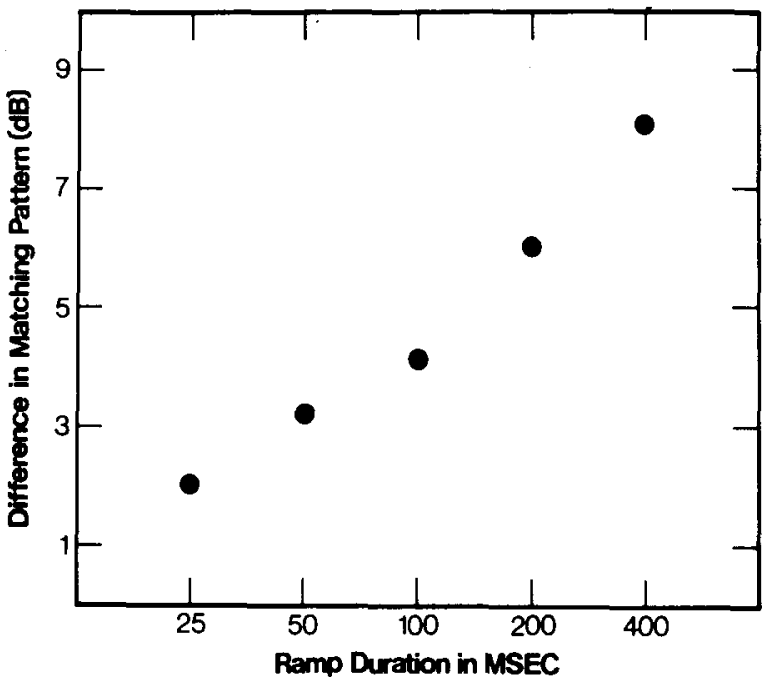

Figure 3. Perceived difference in intensity as a function of ramp duration. Each point is the mean for the two subjects of the estimated effect for a 20-dB intensity-transition range.

transition ranges and estimated the magnitude of the effect for a $20-\mathrm{dB}$ range. The individual data and the estimates are considerably more orderly for the longer ramp duration, where the temporal-order confusions did not occur. ${ }^{2}$ We conclude that the effects of the envelope parameters-intensity-transition range, ramp duration, and constant-intensity interval duration-are roughly monotonic and additive over the ranges we have studied.

\section{EXPERIMENT 2: CLASSIFICATION PROCEDURE}

\section{Method}

Subjects. The two listeners from the first experiment and the first author were the subjects.

Apparatus. The apparatus was generally the same as in the first experiment, except that all three subjects listened simultaneously, in three separate sound-treated rooms.

Stimuli. A single stimulus was presented on each trial. It consisted of an initial and final constant-intensity interval separated by a single transition of the type illustrated in Figure 1. The ramp durations were always equal in a given block of stimuli. Unlike the stimuli in Experiment 1, the two constant-intensity intervals were not always equal in intensity. The stimulus presented on a given trial was selected from a set of possible stimuli that consisted of configurations with initial intensities that differed from final intensities over a range of $\pm 6 \mathrm{~dB}$, in 2-dB steps.

Procedure. A constant-stimulus procedure was used in which the subjects were instructed to indicate after a stimulus presentation whether the initial or final interval was louder. The two constant-intensity intervals were marked with lights.

The principal difficulty involved in using this procedure was in the selection of the stimulus set. When the set was centered on an intensity difference of zero, the data from the first experiment would lead us to predict many more occurrences of one response than of the other. This effect was greatly reduced, however, by the subjects' tendency to use the two responses equally often. One obvious remedy was to center the stimulus set on 


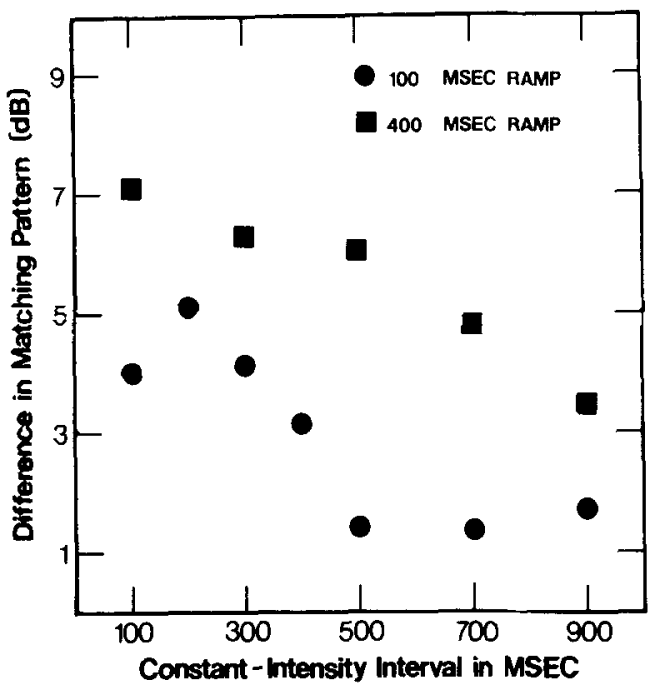

Figure 4. Perceived difference in intensity as a function of the duration of the constant-intensity interval for two different ramp durations.

the intensity difference that was the predicted point of subjective equality (PSE), based on the data from Experiment 1. We rejected this solution because of many studies indicating that subjects' loudness judgments are influenced by the nature of the set of stimuli (e.g., Garner, 1954).

We adopted a compromise procedure in which both types of transitions (an increase in intensity followed by a sudden decrease, and vice versa) were presented at random in a 200 -trial block. The initial set of stimuli consisted of seven stimuli of each transition type, with intensity differences from 6 to $-6 \mathrm{db}$ in 2-dB steps. After every 20 trials, the midpoint of the seven stimuli for each type of transition was moved $2 \mathrm{~dB}$ in the direction required to equalize the number of responses of each kind for each transition. Because the apparatus made it necessary to present the same stimulus to all three subjects, the adjustments were made on the basis of group data, although all of the subsequent analyses were performed on individual data. The net effect of this procedure was that in most cases several points were estimated on each function for each listener and listeners could not tell the size of the effect being measured from their own responses.

After a 200-trial block, the computer counted the number of loud-soft and soft-loud judgments for each intensity difference for each type of transition for each subject. For every category where the percentage of loud-soft judgments was greater than 0 and less than 100 for an individual subiect, the data analysis routine converted the percentage to a $\mathrm{z}$ score. A linear psychometric function was then fit to these $z$ scores, using a ieast-squares criterion. Two psychometric functions were obtained for each subject for each block of trials, one for transitions that increased, then decreased and one for those that decreased, then increased. An example of the two functions is presented in Figure 5.

\section{Results}

The data of interest are the PSEs. They show the difference in intensity required for initial and final intervals to be perceived as equally loud. The difference between the PSEs for the two psychometric functions provides a measure of the magnitude of the illusion that is relatively free from response bias related to interval preference (i.e., a time-order error). ${ }^{3}$ It should also be approximately equal to the loudness-matching measure of the illusion obtained in Experiment 1, which was based on two transitions rather than one.

Mean differences in PSE for different combinations of ramp and constant-intensity interval durations are shown in Figure 6. The conditions are analogous to those in Figure 4, except that shorter constantintensity intervals were dropped and longet intervals added to the set. The correlation between the data points in the two figures for conditions included in both sets is .96 . The mean difference between the two sets of common data points is $0.1 \mathrm{~dB}$.

Having achieved good agreement between the two measures, we used the new measure to explore the effect of larger intensity-transition ranges. Mean differences in PSE as a function of intensity-transition range are shown in Figure 7 . The effect remains linear through ranges as large as $50 \mathrm{~dB}$. The slope of this function (excluding the point at $60 \mathrm{~dB}$ ) is $.24 \mathrm{~dB}$ per decibel increase in transition range. The slope of the equivalent function in Figure 2 is $.22 \mathrm{~dB}$ per decibel. Once again, the agreement between the two procedures is very good. The drop-off above $50 \mathrm{~dB}$ may be an artifact. The sharp 60-dB transition produced annoying clicks that could not be eliminated without altering other parameters. Also, the strategy of beginning with stimuli centered on a difference of zero between the levels of the constant-intensity intervals did not lend itself to the measurement of

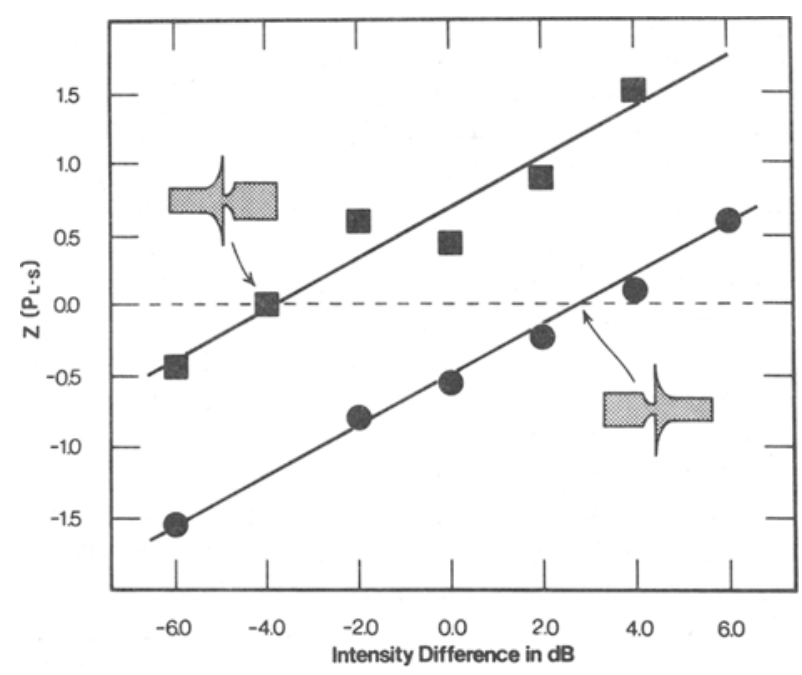

Figure 5. Example of psychometric functions for an individual subject for a condition involving a $1,000-\mathrm{Hz}, 70-\mathrm{dB}$ SPL carrier with a 20-dB transition-intensity range, 100 -msec ramp, and 300 -msec constant-intensity intervals. The ordinate is a normaldeviate transform of the proportion of judgments "loud interval followed by soft interval." The abscissa is the physical difference between the intervals which were separated by the intensity transition. Stimulus configurations are shown for the two points of subjective equality, 


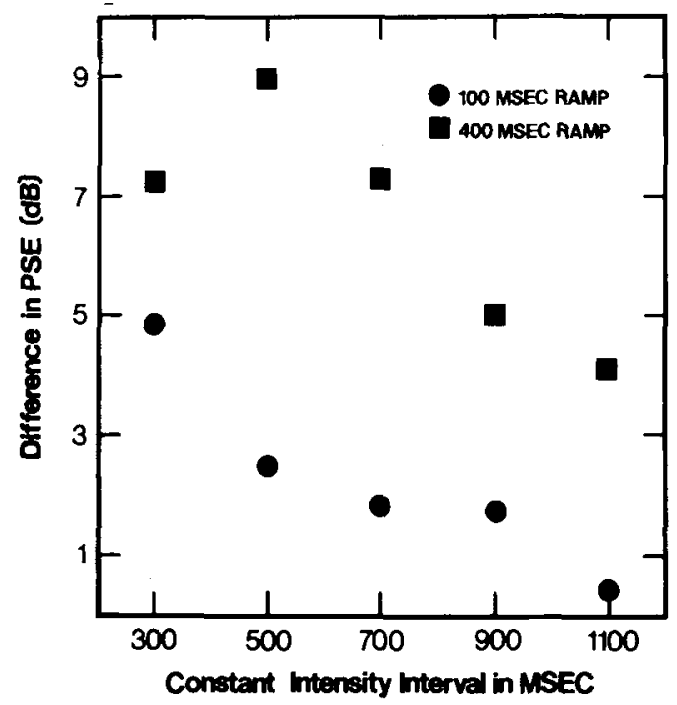

Figure 6. Differences between the points of subjective equality (PSE) for the two forms of the stimulus shown in Figure 5. The data are means for the three subjects, as a function of the duration of the constant-intensity interval for two ramp durations. The carrier was a 70-dB SPL, 1,000-Hz sinusoid, and the intensitytransition range was $20 \mathrm{~dB}$.

large effects. It took too many trials for the procedure to move to the region of interest, and the resulting psychometric functions were very unstable.

\section{DISCUSSION}

Data resulting from two rather different measures of the illusion are in good agreement. With few exceptions, the larger the intensity-transition range and the longer the gradual transition, the larger the effect. The effect decays over time, growing smaller for longer constant-intensity intervals. We did not attempt to measure the maximum duration, but observed effects greater than $12 \mathrm{~dB}$ for short constantintensity intervals and effects greater than $4 \mathrm{~dB}$ lasting $1,100 \mathrm{msec}$. These effects were obtained using combinations of parameters that were, in retrospect, clearly not optimum.

Producing and measuring the illusion is considerably easier than explaining it. Two hypotheses that come to mind are: (1) The subjects are integrating energy across the transition intervals as well as the constant-intensity intervals in generating loudness estimates, and (2) the phenomenon may be related to loudness enhancement (e.g., Elmasian \& Galambos, 1975; Irwin \& Zwislocki, 1971). The hypothesis that subjects are listening at the wrong time cannot account for the size of the effects observed. In most cases, perfect integration across the peaks and troughs surrounding the constant-intensity intervals would not result in an energy difference large enough to produce the differences in loudness that were meas- ured. Also, the durations involved are longer than the 150-200-msec maximum integration time observed in experiments where loudness was measured as a function of duration (Stevens \& Hall, 1966). Finally, if we assume that subjects err in when they listen by the same amount across all conditions, we would predict a larger effect in conditions where the ramp duration was shorter and the energy difference was greater. The opposite was observed.

The loudness-enhancement hypothesis is more difficult to refute, but would not constitute an explanation in any case. The effects we measured differed from those observed in loudness-enhancement experiments in that they were roughly symmetric. In the matching paradigm, we tried a condition comparable to contralateral loudness enhancement, in which the transition portions of the test stimulus were presented to one ear and the constant-intensity intervals were presented to the other, and observed no effect. We cannot account for the illusion, then, in terms of familiar auditory phenomena.

The brightness analogue of the illusion has received a great deal of attention in recent years (Arend, Buehler, \& Lockhead, 1971; Campbell, Howell, \& Robson, 1971; Cornsweet, 1970; Davidson \& Whiteside, 1971; Land \& McCann, 1971; Shiffman \& Crovitz, 1972), and this literature contains several related explanations of the effect. The two accounts that are most applicable to the loudness illusion are the discussion by Cornsweet (1970) and a two-stage model of brightness proposed by Shiffman and Crovitz (1972).

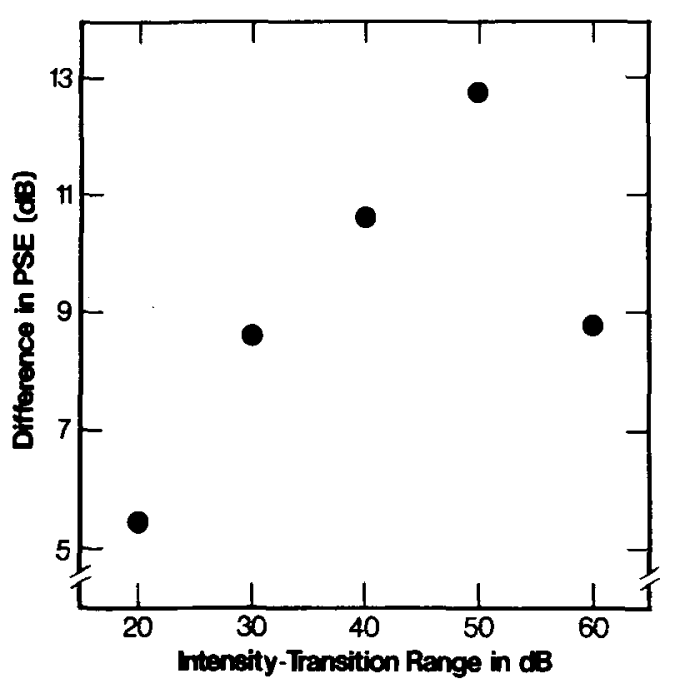

Figure 7. Mean difference in the points of subjective equality (PSE) for the three subjects as a function of intensity-transition range. The carrier was a 50-dB SPL, 1,000-Hz sinusoid. The ramp duration was $100 \mathrm{msec}$, and the constant-intensity interval was 300 msec. 
Cornsweet $(1970$, p. 346) points out that the spatial-frequency components for a step function in luminance (an edge or a square wave) differ from the components for a double-discontinuity function (the luminance function in the Craik-O'BrienCornsweet illusion) only at the low frequencies. $\mathrm{He}$ argues that these two stimuli should look alike because the visual system attenuates low spatial frequencies. His analysis predicts, however, that both stimuli should be seen as sharp discontinuities in an otherwise uniform field, and he is unable to explain why they are both seen as edges or bars.

In a similar vein, Campbell et al. (1971) note that the double-discontinuity luminance function is equivalent to a square wave with the fundamental spatial frequency subtracted out. We continue to see a square wave, filling in the "missing fundamental," just as we hear a missing fundamental in experiments on pitch perception (e.g., Schouten, Ritsma, \& Cardozo, 1962).

Shiffman and Crovitz (1972) present a linearsystems analysis similar to Cornsweet's, but in the space domain rather than the frequency domain. They describe the system in terms of the impulseresponse function rather than the modulationtransfer function. The first stage of their model consists of a spatial filter with a double-difference, impulse-response function modeled on the function describing lateral inhibition on the retina (Ratliff, 1965). The filter input is the luminance function on the retina. The output is a neural-response function containing information only about the second derivatives of the input. As in Cornsweet's analysis, this output is the same for both a step-function or doublediscontinuity input. Shiffman and Crovitz assume a second stage in which this output is integrated to produce a brightness function. They assume that the nature of the integration is such that the final output for a step function in luminance will be a step function in brightness. The net effect is that a double discontinuity in luminance is also perceived as a step function in brightness.

A similar auditory model could be developed for the Rawdon-Smith illusion. The situation is not as simple as it is for the visual analogue, however, and we are unable to specify an impulse-response function on the basis of the data reported here. The data show a nonlinear relation between the input function and the difference in apparent intensities. A 10-dB increase in transition range, for example, results in only a $2.4-\mathrm{dB}$ change in the apparent intensities. It could be that the form of the test stimulus was not optimal or that the system itself is nonlinear. The most impressive feature of the analogous visual illusion is that a local discontinuity produces global effects. Brightness is altered over the entire field to the left and right of the discontinuity. The data for

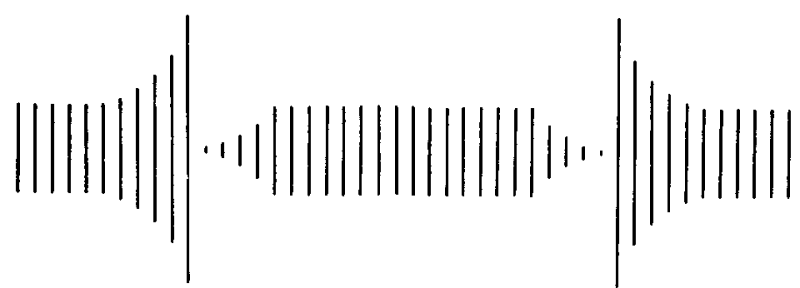

Figure 8. An example of a line-length illusion reported by Crovitz (1976). The pattern shown here is analogous to an auditory pattern with a constant-intensity interval three times the length of the slow transitions (as in the 300 -msec 100 -msec condition that appears in many of the figures) and an intensity-transition range of $20 \mathrm{~dB}$. Unlike the Craik-O'Brien illusion, the line-length illusion is a function of spatial frequency and appears stronger when viewed from a greater distance.

the auditory illusion as a function of constantintensity interval duration indicate that the illusion decays over time. In spite of these difficulties, the auditory illusion may be a rich source of information regarding the dynamic aspects of the system.

We have emphasized the analogy between the illusion involving brightness at different points in space and the illusion involving loudness at different points in time. Several other variations exist, however, and should be noted. We have found, in informal observations, that, if we modulate frequency instead of intensity, we can produce a pitch difference instead of a loudness difference. The pitch illusion is not as strong, however, as the illusions reported by Risset (1969) or Shepard (1964). Variations also exist for the visual illusion. Anstis (1967) and Walker (1974) have reported one in which luminance is varied over time rather than over space. MacKay (1973) has reported a variation in which spatial frequency is modulated with a double-difference pattern so that equally spaced vertical bars look closer together at some points and farther apart at others. ${ }^{4}$ Finally, Crovitz (1976) has reported an analogous illusion in line length. An example of this illusion is shown in Figure 8, using the same configuration we used in Experiment 1 . The physical length of the lines represents the physical amplitude of waveforms we used, and the apparent length of the lines represents the apparent intensity. The number of these similar phenomena suggests that the illusion is pervasive and probably related to basic properties of sensory systems.

\section{REFERENCES}

ANstis, S. M. Visual adaptations to gradual changes of intensity. Science, 1967, 155, 710-712.

Arend, L. E.. Buehler, J. N., \& Lockhead, G. R. Difference information in brightness perception. Perception \& Psychophysics. 1971, 9. 367-370.

Camprell, F. W., Howell, E. R., \& Robson, J. G. The appearance of gratings with and without fundamental Fourier component. Joumal of Physiology, 1971, 217, 17-18. 
Cornsweet, T. N. Visual perception. New York: Academic Press, 1970.

CRAIK, K. J. W. Visual adaptation. PhD thesis, University of Cambridge, 1940.

Crovitz, H. F. Perceived length and the Craik-O'Brien illusion. Vision Research, 1976, 16, 435.

Davidson, M., \& Whiteside, J. A. Human brightness perception near sharp contours. Journal of the Optical Society of America, 1971, 61, 530-536.

Elmasian, R., \& Galambos, R. Loudness enhancement: Monaural, binaural, and dichotic. Journal of the Acoustical Society of America, 1975, 58, 229-234.

GARNER, W. R. A technique and a scale for loudness measurement. Journal of the Acoustical Society of America, 1954, 26. $73-88$.

IRwIN, R. J., \& ZwISLOCKI, J. J. Loudness effects in pairs of tone bursts. Perception \& Psychophysics, 1971, 10, 189-192.

Jesteadt, W., Green, D. M., \& WIER, C. C. Auditory analog to the Craik-O'Brien-Cornsweet illusion in vision. Journal of the Acoustical Society of America, 1976, 59, S83(A).

LAND, E. H., \& MCCANN, J. J. Lightness and retinex theory. Journal of the Optical Society of America, 1971, 61, 1-11.

MACKAY, D. M. Lateral inhibition between neural channels. sensitive to texture density? Nature, 1973, 245, 159-161.

O'Brien, V. Contour perception, illusion and reality. Journal of the Optical Society of America, 1958, 48, 112-119.

RATLIFF, F. Mach bands: Quantitative studies on neural networks in the retina. San Francisco: Holden-Day, 1965.

Rawdon-Smith, A. F., \& Grindeey, G. C. An illusion in the perception of loudness. British Journal of Psychology, 1935. 26, 191-195.

Risset, J. C. Pitch control and pitch paradoxes demonstrated with computer-synthesized sounds. Journal of the Acoustical Society of America, 1969, 46, 88(A).

Schouten, J. R., Ritsma, R. J., \& Cardozo, B. L. Pitch of the residue. Journal of the Acoustical Society of America, $1962,34,1418-1424$.

SHEPARD, R. N. Circularity in judgments of relative pitch. Journal of the Acoustical Socity of America, 1964, 36, 2346-2353.

SHERwOOd, S. L. (Ed.) The nature of psychology: A selection of papers, essays and other writings by the late Kenneth J. W. Craik. Cambridge, England: The University Press, 1966.
Shiffman, H., \& Crovitz, H. F. A two-stage model of brightness. Vision Research, 1972, 12, 2121-2131.

Stevens, J. C., \& Hall, J. W. Brightness and loudness as functions of stimulus duration. Perception \& Psychophysics, 1966, 1, 319-327.

W ALKER, J. T. A new rotating gradient disk: Brightness, flicker, and brightness after effects. Vision Research, 1974, 14, 223-228.

\section{NOTES}

1. Subjects did not always perceive the initial and final constantintensity intervals in the test stimulus as being equal in loudness. They were instructed in that case to concentrate on the middle and final intervals. The initial interval was included to avoid the occurrence of a sudden onset followed immediately by a transition. We then found it necessary to include a corresponding interval in the matching stimulus to avoid confusion about which intervals were to be matched.

2. Data for the condition involving a $400-\mathrm{msec}$ ramp and 300 -msec constant-intensity interval occur in both Figures 3 and 4 . The condition was tested twice and the two points in the figures represent independent estimates of the effect.

3. Rawdon-Smith and Grindley (1935) suggest that the illusion is asymmetric, that a transition in one direction produces a larger effect than a transition in the other. That effect would be confounded with order effects in the present procedure. Although the points of subjective equality were rarely symmetric around zero, there was no consistent pattern in the asymmetries for the three subjects.

4. MacKay (1973) noted that it was possible to quantify the illusion he described by determining what compensation was required to nullify it. That is, of course, the essence of the second measurement procedure we describe. Mackay did not present any data. To our knowledge, no parametric studies have been done on conditions influencing the magnitude of any of the analogous visual illusions.

(Received for publication August 8, 1977; revision accepted January 9, 1978.) 